\title{
Detailed study of the direct numerical observation of the Kramers turnover in the $\mathrm{LiNC} \rightleftharpoons \mathrm{LiCN}$ isomerization rate
}

\author{
P. L. García Müller, ${ }^{1}$ Rigoberto Hernandez, ${ }^{2}$ R. M. Benito, ${ }^{3}$ and F. Borondo ${ }^{4}$ \\ ${ }^{1}$ Centro de Investigaciones Energéticas Medioambientales y Tecnológicas, Avda. Complutense 40, \\ 28040 Madrid, Spain \\ ${ }^{2}$ Center for Computational Molecular Sciences and Technology, School of Chemistry and Biochemistry, \\ Georgia Institute of Technology, Atlanta, Georgia 30332-0430, USA \\ ${ }^{3}$ Grupo de Sistemas Complejos and Departamento de Física y Mecánica, Escuela Técnica Superior \\ de Ingenieros Agrónomos, Universidad Politécnica de Madrid, 28040 Madrid, Spain \\ ${ }^{4}$ Departamento Química, and Instituto de Ciencias Matemáticas (ICMAT), Universidad Autónoma de Madrid, \\ Cantoblanco, 28049 Madrid, Spain
}

(Received 6 August 2012; accepted 22 October 2012; published online 27 November 2012)

\begin{abstract}
According to Kramers, rates of molecular process are expected to follow a rise and fall from low friction (at little to no interaction with the environment) to high friction (at typical liquid densities and above). This so-called Kramers turnover was recently observed and delineated in the case of the $\mathrm{LiNC} \rightleftharpoons \mathrm{LiCN}$ isomerization reaction in the presence of an argon bath [P. García-Müller, R. Hernandez, R. M. Benito, and F. Borondo, Phys. Rev. Lett. 101, 178302 (2008)]. The rates were obtained using direct molecular dynamics of an all-atom representation and the Langevin dynamics of a projected representation. We now provide further evidence that the forward and backward rates are indeed exhibiting the turnover. The rates are also seen to agree remarkably well with the Pollak-Grabert-Hänggi rate formulas in regimes satisfying the theory underlying assumptions. At higher temperatures, when the theory is expected to fail, the solvated LiCN isomerization continues to exhibit activated dynamics following the turnover. (C) 2012 American Institute of Physics. [http://dx.doi.org/10.1063/1.4766257]
\end{abstract}

\section{INTRODUCTION}

Brown ${ }^{1}$ first observed that the seemingly random motion of small particles suspended in a liquid are governed not by the details of the solvent particle motions but by their collective actions. The details of the solvent-particle interaction are surmised through statistical properties of the response, e.g., the mean-field friction and the thermal fluctuations. The Langevin equation makes these connections concrete. Therein, it is clear that no atomistic details of the solvent are necessary. It relies instead only on the statistical properties of the solvent-particle interaction. The rate of population decay of an activated particle from a reactant region, as governed by this equation, generally decreases with increasing interaction-vis-a-vis coupling or friction-to the solvent in the so-called high-friction limit. This limit conforms to our typical experience in which rates are slowed by increasing friction. However, Kramers ${ }^{2}$ observed that in the so-called low-friction limit, rates would actually go up with increasing friction. The two limits taken together give rise to the Kramers turnover in the rate. It is bounded from above by the transition state theory rate, and the turnover behavior has been captured beautifully by theory over the entire domain. ${ }^{3,4}$

In the context of chemical reaction rate theory, ${ }^{5-11}$ an elusive question has centered on the existence (or lack thereof) of chemical reactions which give rise to increasing rates in the low-friction regime and thereby a true Kramers turnover. Reports observing apparent increases in the rates with friction include the isomerization of trans- stilbene, ${ }^{12,13}$ isomerization of cyclohexane,${ }^{14}$ internal rotation of $N, N$-dimethyltrichloroacetamide ${ }^{15}$ the isomerization of $\mathrm{CH}_{3}-\mathrm{CN} \rightleftharpoons \mathrm{CN}-\mathrm{CH}_{3},{ }^{16}$ and the degradation of $\beta$-carotene. ${ }^{17}$ Unfortunately, these have not been entirely conclusive because the solvent conditions allowed for unaccounted changes in temperature, pressure, or other factors, and not just the friction. Such changes have long been known to give rise to apparent turnovers. ${ }^{18}$

In recent work, ${ }^{19}$ we suggested that the $\mathrm{LiCN}$ isomerization reaction in the presence of an argon bath could give rise to an observable Kramers turnover. This all-atom simulation is complementary to coarse-grained models, such as the one recently reported by Perkins et al. ${ }^{20}$ on the antigauche isomerization in butane. In our work, we varied the density of the argon bath which in turn varied the effective interaction-namely, the friction - between the bath and the dynamics of the isomerization. The rates were obtained at sufficiently low friction that direct dynamics could be computed. The rates followed the rise and fall of the Kramers turnover and were surmised by the corresponding PollakGrabert-Hänggi (PGH) turnover theory despite being only weakly in the activated regime. In this article, we go further in elaborating the earlier results of Ref. 19. We establish the relation between the friction and density and confirm the temperature stability. We explore the degree to which the dynamics satisfies the criteria for PGH to hold. In so doing, we believe that we establish the fact that this system is indeed exhibiting a true Kramers turnover for a chemical system. 
The organization of this article is as follows: In Sec. II, an atomistic description of the chemical reaction being studied is outlined. In Sec. III, the molecular dynamics simulations of the reaction rates are compared to the PGH theory. Finally, the results from theory and numerical experiments are discussed in Sec. IV.

\section{SYSTEM}

The isomerization between the $\mathrm{LiNC}$ and $\mathrm{LiCN}$ structures-with the ordering of the atoms indicating the bonded structure-is known to be activated through bimolecular collisions, as represented by the reaction

$$
\mathrm{Li}-\mathrm{NC}+\mathrm{Ar} \underset{k_{b}}{\stackrel{k_{f}}{\rightleftharpoons}} \mathrm{NC}-\mathrm{Li}+\mathrm{Ar} .
$$

In the presence of an argon bath these collisions are frequent. As indicated in Sec. III B below, for the typical temperatures of this study, one to three collisions suffice to overcome the activation barrier in the forward or backward direction, respectively. The friction felt by the reaction system as a variation of the density involves many more collisions than this. Consequently, the primary effect of the variation of the density of the argon bath (in this relatively condensed regime) on the isomerization rate is through the friction constant. The rates are obtained at an all-atom-or microscopic level-by means of classical molecular dynamics (MD) simulations, solving the Newtonian dynamics of all the atoms interacting in the system.

Lithium cyanide, $\mathrm{LiNC} / \mathrm{LiCN}$, is a triatomic molecule with three internal degrees of freedom. Its vibrational motions can be described in terms of the Jacobi coordinates indicated in Fig. 1(a): the distance $R$ between the Li atom and the center of mass of the $\mathrm{C} \equiv \mathrm{N}$ bond; the interatomic distance $r$ between the $\mathrm{C}$ and $\mathrm{N}$ atoms; and the bending angle $\psi$ between the $\mathrm{C} \equiv \mathrm{N}$ bond axis and the $R$ direction. The $\mathrm{C} \equiv \mathrm{N}$ vibrational

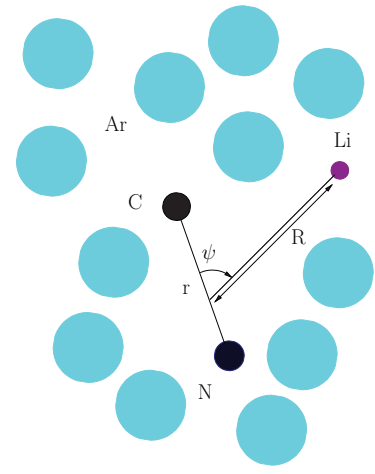

(a)

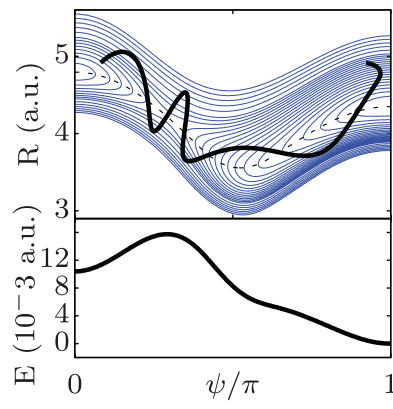

(b)
FIG. 1. Description of the LiNC/LiCN isomerizing system embedded in a bath of Ar atoms: (a) definition of the Jacobi coordinates for the molecule; (b) contour plot of the internal molecular potential (top). The dashed curve corresponds to the minimum energy path connecting the two isomer wells, whose energy profile is shown in the bottom panel. Equipotential curves start from 0.001 a.u. in the $\operatorname{LiNC}$ well $(\psi=\pi)$ with 0.001 a.u. steps on each isomer well. Open equipotentials, connecting both isomers, are drawn at increments of 0.002 a.u. An example of a reactive trajectory that initially starts in the reactant well and crosses the free energy barrier to arrive at the product well is also shown superimposed.
TABLE I. Numerical values (in a.u.) of the parameters in the Lennard-Jones, $V_{i j}=4 \epsilon\left[\left(\sigma / r_{i j}\right)^{12}-\left(\sigma / r_{i j}\right)^{6}\right]$, and Born-Mayer, $V_{i j}=A \exp \left(-r_{i j} / r_{0}\right)$, potentials used in Eq. (1).

\begin{tabular}{lccl}
\hline \hline Pair & Potential & $\sigma$ or $r_{0}$ & \multicolumn{1}{c}{$\epsilon$ or $A$} \\
\hline Ar-Ar & LJ & 6.265 & $4.594 \times 10^{-4}$ \\
Ar-N & LJ & 5.724 & $2.220 \times 10^{-4}$ \\
Ar-C & LJ & 5.724 & $2.220 \times 10^{-4}$ \\
Ar-Li & BM & 1.923 & 92.537 \\
\hline \hline
\end{tabular}

frequency is much higher than that for the other molecular modes, and thus $r$ can be approximately frozen at its equilibrium value, $r_{e}$, during long enough time scales. Taking this mode to be frozen reduces the internal dynamics to two internal degrees of freedom, $R$ and $\psi$.

The potential energy surface (PES) in these two variables was fit to $a b$ initio electronic structure calculations by Essers et al. ${ }^{21}$ and is illustrated as a contour plot in Fig. 1(b). The PES contains two potential wells corresponding to the two linear isomers: $\mathrm{LiCN}$ at $\psi=0$, and $\operatorname{LiNC}$ at $\psi=\pi$, that is the most stable one. They are separated by an energy barriers of 0.0159 a.u. in the direction of the forward reaction $\mathrm{LiNC} \rightarrow \mathrm{LiCN}$, and 0.005 a.u. in the direction of the backward reaction, $\mathrm{LiCN} \rightarrow \mathrm{LiNC}$. The minimum energy path connecting the two isomer wells has been drawn superimposed as a dashed line over the contours of Fig. 1(b) (top). The energy profile along this line is shown in Fig. 1(b) (bottom).

As the LiNC/LiCN molecule is embedded in the bath, the total potential energy of the system will be the sum of the internal energy of the LiNC/LiCN molecular system, $V_{\mathrm{int}}^{\mathrm{LiNC} / \mathrm{LiCN}}$, the internal energy of the argon bath, i.e., the interaction between Ar atoms, $V_{i j}^{\mathrm{Ar}-\mathrm{Ar}}$, and the two body interactions between the $\mathrm{Ar}$ and the atoms in the molecule, $V_{j}^{\mathrm{Ar}-\mathrm{X}}$,

$$
\begin{aligned}
V= & V_{\mathrm{int}}^{\mathrm{LiNC} / \mathrm{LiCN}}+\lambda g+\sum_{i>j} \sum_{j} V_{i j}^{\mathrm{Ar}-\mathrm{Ar}} \\
& +\sum_{j}\left(V_{j}^{\mathrm{Ar}-\mathrm{Li}}+V_{j}^{\mathrm{Ar}-\mathrm{C}}+V_{j}^{\mathrm{Ar}-\mathrm{N}}\right),
\end{aligned}
$$

where $V^{\mathrm{Ar}-\mathrm{X}}(\mathrm{X}=\mathrm{Ar}, \mathrm{C}, \mathrm{N}, \mathrm{Li})$ are described by the standard pairwise Lennard-Jones (LJ) and Born-Mayer (BM) potentials, parameterized in the usual way; the details are summarized in Table I. As can be seen, an extra term, $\lambda g$, has been added to the internal potential. Its purpose is to keep the $\mathrm{C}-\mathrm{N}$ distance fixed by counteracting, through the Laplace multiplier $\lambda$, the effect of the bath atoms on $\mathrm{C}-$ $\mathrm{N}$ coordinate. Full details of this procedure will be given elsewhere.

\section{NUMERICAL OBSERVATION OF THE KRAMERS TURNOVER}

\section{A. Molecular dynamics}

\section{Methodology}

In our model, the LiNC/LiCN molecule is embedded in a bath of Ar atoms whose average interatomic distance ranges 
from 5 to 30 a.u. depending on the specific density. The propagation of the MD is performed using in-house codes capable of integrating Lagrange equations of motion for all the atoms using Verlet algorithm, periodic boundary conditions, and interaction lists. Imposing periodic boundary conditions allows the center of the interaction cell to be fixed at the center of mass of the molecule at each step, without loss of generality. A set of $512 \mathrm{Ar}$ atoms have been included in the periodic box representing the system. The corresponding canonical ensemble is sampled by setting the (reduced) thermal energies (relative to the barrier height, $V^{+} / k_{\mathrm{B}} T$ ) to 1.6 and 0.4 for the forward and backward reactions, respectively. The temperature is established during the initialization phase wherein the Ar velocities are rescaled at each step so that the overall average kinetic energy matches the equilibration temperature. Thereafter, during the calculation phase, the system is propagated without thermostats or temperature rescalings.

At the elevated temperatures explored here, trajectories cross between reactants and products sufficiently frequently that one does not need to use rate formula based on rare events. Instead, the rates are obtained directly by monitoring the flux of trajectories through the dividing surface, and keeping $\log$ of the trajectories that are initially in the reactant well and end up in the product region after a sufficiently long time. The reaction coordinate is dominated (tracked) by motion along $\psi$, the bending $\mathrm{Li}-\mathrm{C}-\mathrm{N}$ mode. Specifically, the rate is computed through a best fit procedure to the first order reaction expression

$$
\log N(t)=-k t+N(0),
$$

where $N(t)$ is the population of the reactants at time $t$, and $k$ is the corresponding reaction rate. In Fig. 1(b), we have plotted the PES overlaid by a typical reactive trajectory, that initially starts in the reactant well and crosses the free energy barrier to arrive at the product well after a sufficiently long time. Such trajectories contribute only once to the flux regardless of the number of barrier recrossings they encounter, and hence our calculation is formally exact.

\section{Analysis of numerics}

Forward, $k_{f}$, and backward, $k_{b}$, reaction rates were calculated at several bath densities, ranging from 0.5 to $90 \mathrm{~mol} / \mathrm{l}$, by fitting the reactant lifetime distributions to an exponential decay law by simple linear regression. The procedure is illustrated in Fig. 2, where two representative examples for the forward reaction at different values of the bath density are shown. For the lower value of the density (top panel), a number of trajectories that quickly dissociate at short times $\left(t \lesssim 2 \times 10^{4}\right.$ a.u. $)$ are seen, these deviating from the exponential decay that is observed at longer times. These ballistic trajectories result from the initial nonequilibrium distribution and are, therefore, not taken into account in the computation of the reaction rates. This kind of "anomalous" trajectories do not occur, on the other hand, at higher values of the density (see lower panel in the figure). Semilog plots of $N_{\mathrm{LiNC}} \rightarrow \mathrm{LiCN}$ vs. time, from which $k_{f}$ can be obtained, are shown at the insets.
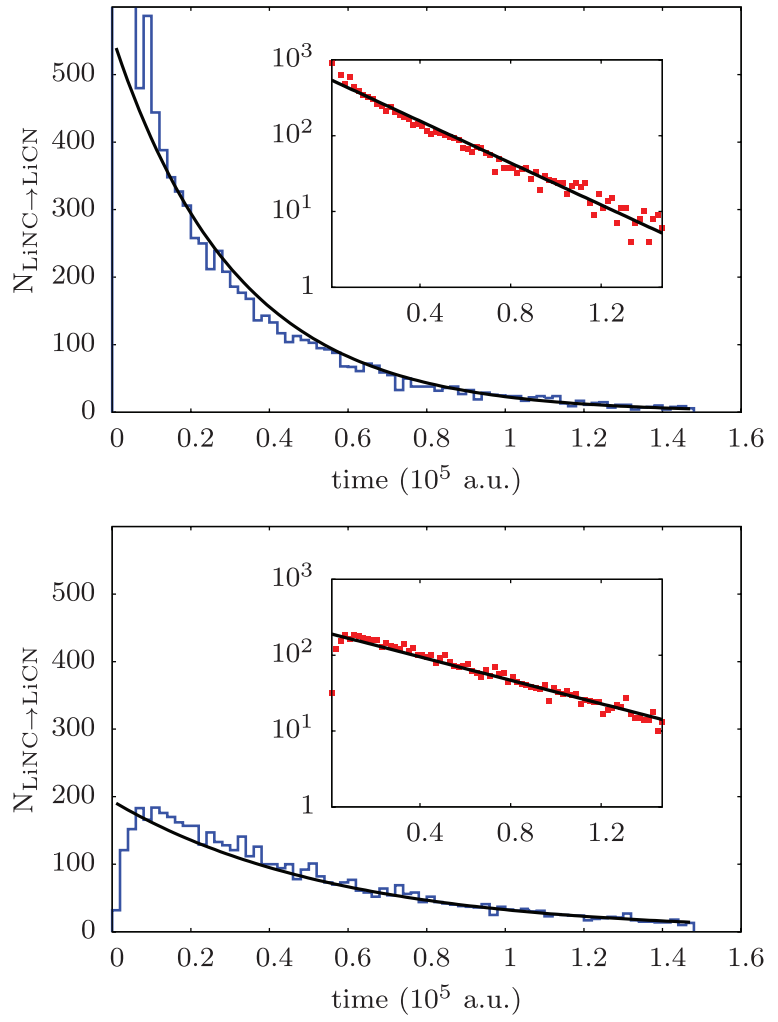

FIG. 2. Reactant lifetime distributions calculated by molecular dynamics for $\mathrm{LiNC} \rightarrow \mathrm{LiCN}$ in an Ar bath at $T=5500 \mathrm{~K}$ and two different values of the bath density: $11.2 \mathrm{~mol} / \mathrm{l}$ (top) and $89.66 \mathrm{~mol} / \mathrm{l}$ (bottom). The corresponding semilog plots of the distributions, from which the reaction rates are obtained, are shown in the insets.

The bath friction kernel has also been calculated from the autocorrelation of the forces exerted on the molecule center of mass. Once this magnitude is known, the bath viscosity is readily obtained as the zero frequency Laplace transform. This is an exact relationship if the friction kernel is exponentially decaying, and approximate otherwise. The corresponding results are shown in Fig. 3. Clearly, the friction is changing with density, and both effects must be considered when establishing the reaction rate dependence on friction, as will

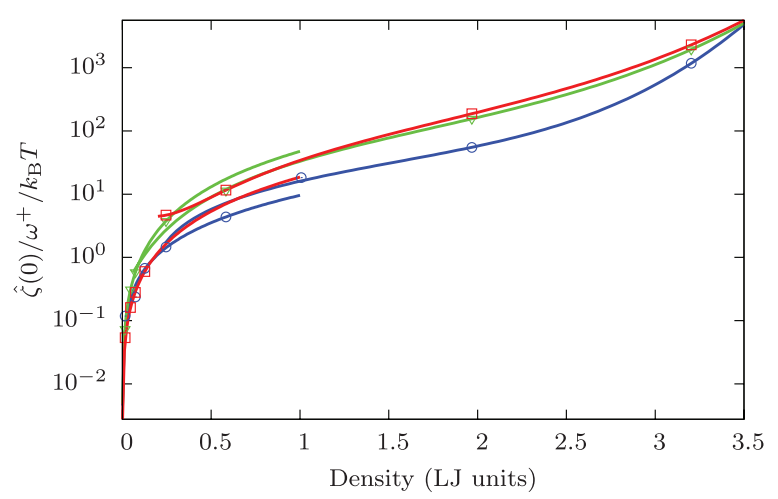

FIG. 3. Viscosity calculated as the zero frequency Laplace transform of the friction kernel (normalized to the bath temperature and barrier frequency) as a function of the bath density (in Ar-Ar Lennard-Jones units) for three different values of the temperature: $T=1500 \mathrm{~K}$ (blue circles), $3500 \mathrm{~K}$ (green inverse triangles), and $5500 \mathrm{~K}$ (red squares). The low and high density fits to expression (3) using the coefficients of Table II are also shown in solid lines. 
be discussed below. Figure 3 clearly shows how the viscosity scales with the bath density. A slope change can be identified at a density of around $0.7 \mathrm{LJ}$ units (or $30 \mathrm{~mol} / \mathrm{l}$ ), corresponding to a mean interatomic distance of $0.38 \mathrm{~nm}$. This is very close to the $r_{\min }$ distance of the LJ potential for Ar$\operatorname{Ar}\left(r_{\min }=0.37 \mathrm{~nm}\right)$. Therefore, the bath is likely in liquid or supercritical phase below this density at the temperatures considered here. At the highest densities, the systems become close-packed with mean interatomic distances values down to $0.29 \mathrm{~nm}$, well below $\sigma^{\mathrm{Ar}-\mathrm{Ar}}=0.33 \mathrm{~nm}$ ).

In order to better understand how the friction depends on viscosity, we compare the values in our calculation with those previously found by Straub et al. ${ }^{22}$ These authors studied a LJ system with parameters similar to those of the Ar bath in the present work, after the relative values of $\sigma$ and $\epsilon$ are scaled. The normalized viscosity as a function of the density can be well approximated by the expression ${ }^{22}$

$$
\frac{\hat{\zeta}(0)}{\omega^{+} k_{\mathrm{B}} T}=a \rho+b \rho^{2} \mathrm{e}^{\mathrm{c} \rho^{4}}
$$

where only the coefficients $a$ and $b$ are considered in the low density region, i.e., $c_{\mathrm{L}}=0$. Best fits of Eq. (3) to the computed viscosity of the Ar bath are shown in Fig. 3, and the corresponding parameters $a, b$, and $c$ are reported in Table II. It should be noted that the main differences between the present numerical analysis and that in Ref. 22 are:

1. In Ref. 22, the viscosity is derived from the Einstein relation, $\hat{\zeta}(0)=k_{\mathrm{B}} T / D$, and the diffusion coefficient, $D$, is derived from the mean square displacement of the particles.

2. The reduced temperatures $T^{*}\left(=k_{\mathrm{B}} T / \epsilon^{\mathrm{Ar}-\mathrm{Ar}}\right)$ considered in this work, namely, 10.34, 24.13, and 37.93, are much higher than those used in Ref. 22 (at values near 2.5).

3. The high viscosity regime appears to be flattened out in the MD data, $\left(c_{\mathrm{H}}\right.$ is nearly two orders of magnitude lower than in Ref. 22). This seems to be consistent with the fact that the interaction is limited by the total number of particles that can fit within the solvent shell(s), and this number eventually reaches a maximum.

Only at the lowest temperature and in the low density regime is the $a / b$ ratio $(=0.91)$ in direct agreement with the result (=1.03) of Ref. 22. However, the latter did not report the higher density regime observed here, and the observed differences are consistent with the theoretical expectations, as discussed above.

TABLE II. Best fit coefficients of the bath viscosity [see Eq. (3)] as a function of the density for three different values of the temperature, in the low (L) and high $(\mathrm{H})$ density approximations. Notice that $c_{\mathrm{L}}=0$.

\begin{tabular}{rcrrrc}
\hline \hline$T(\mathrm{~K})$ & $a_{\mathrm{L}}$ & \multicolumn{1}{c}{$b_{\mathrm{L}}$} & \multicolumn{1}{c}{$a_{\mathrm{H}}$} & \multicolumn{1}{c}{$b_{\mathrm{H}}$} & $c_{\mathrm{H}}$ \\
\hline 1500 & 4.55 & 5.02 & 11.55 & 5.73 & 0.028 \\
3500 & 4.81 & 42.64 & 2.34 & 29.87 & 0.017 \\
5500 & 2.75 & 15.89 & -15.17 & 42.95 & 0.016 \\
\hline \hline
\end{tabular}

\section{B. Pollak-Grabert-Hänggi theory}

\section{Theory}

The resulting reaction rates in the $\mathrm{LiNC} \rightleftharpoons \mathrm{LiCN}$ isomerization simulations observed in Sec. III A can be compared directly to the $\mathrm{PGH}^{4}$ theory predictions. In order to carry out this comparison, a reduced-dimensionality potential approximation model is required. For this purpose, we have used the Straub-Borkovec-Berne (SBB) potential model. ${ }^{23}$ This choice imposes the additional assumption that the potential can be described approximately as a series of merged parabolas. The PGH rates have been calculated for each set of conditions, both with respect to the potential of the mean force (PMF), and also for the bare potential to have a reference for comparison.

The effective subsystem is constrained to the minimum energy path $\vec{x}_{\text {MEP }}$ (MEP, dashed black curve in Fig. 1(b)) connecting both isomer wells. It lies on the two-dimensional space $\vec{x} \equiv(R, \psi)$ characterizing the position of Li relative to the $\mathrm{CN}$ center of mass. As shown in the figure, the path can be parameterized by the angle, $\psi$, and written as $R_{\mathrm{MEP}}(\psi)$. This, in turn, allows us to represent the bare Hamiltonian along the minimum energy path as

$$
\mathcal{H}(\psi)=\frac{p_{\psi}^{2}}{2 I_{\psi}}+V_{\mathrm{MEP}}(\psi)
$$

where we define $V_{\mathrm{MEP}}(\psi) \equiv V\left(\vec{x}_{\mathrm{MEP}}(\psi)\right.$ ) (black curve in Fig. 4) using the interaction potential $V$ described in Eq. (1). The effective moment of inertia (necessary for the kinetic energy computation) is set to

$$
\frac{1}{I_{\psi}}=\frac{1}{\mu_{L i, C N} R(\psi)^{2}}+\frac{1}{\mu_{C, N} r_{0}^{2}},
$$

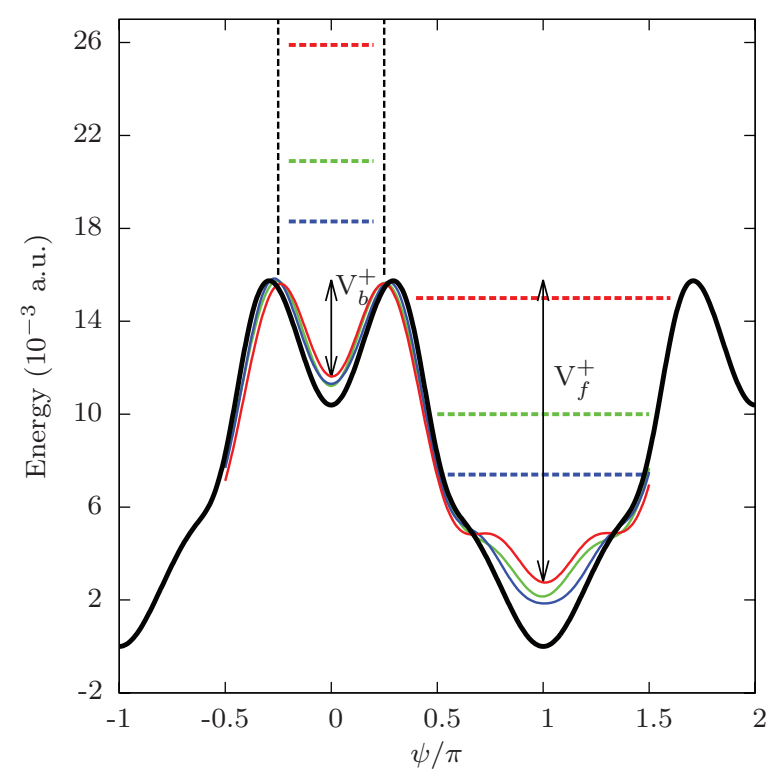

FIG. 4. Bare (black solid line) and PMF (noise FFT filtered) potentials for three different values of the temperature: $T=2500 \mathrm{~K}$ (blue solid line), $3500 \mathrm{~K}$ (green solid line), and $5500 \mathrm{~K}$ (red solid line). The corresponding thermal energies: $k_{\mathrm{B}} T=0.0074,0.0100$, and 0.0158 a.u., respectively, have also been plotted as horizontal lines with the same colors. 
where $\mu_{A, B}$ is the reduced mass of the pair of total masses for the corresponding particles $A$ and $B$, respectively. The distances $R(\psi)$ and $r_{0}$ correspond to that between $\mathrm{Li}$ and the center of mass of $\mathrm{CN}$ and that for the $\mathrm{CN}$ bond distance, respectively. The latter is fixed to be the minimum energy configuration, whereas the former depends on the extension of $\mathrm{Li}$ as a function of $\psi$. This representation is effectively an approximation of Miller's reaction path Hamiltonian ${ }^{24}$ by limiting the curvature effects to be included through the $R$ dependence in the moment of inertia. The one-dimensional potential along this reaction coordinate serves as the bare potential in the Langevin equation. The PMF (colored curves in Fig. 4) is determined from the Boltzmann weighted average ${ }^{25}$

$$
V_{\mathrm{PMF}}(\psi)=-k_{\mathrm{B}} T \ln \int d \vec{q} \delta[\psi(\vec{q})-\psi] e^{-V(\vec{q}) / k_{\mathrm{B}} T},
$$

where $\vec{q}$ denotes the Cartesian coordinates of all the atoms in the system, and $\psi(\vec{q})$ denotes a function extracting the $\psi$ component from this multidimensional position vector. As this PMF accounts for only one of the two symmetrically identical open channels to reaction, the $\mathrm{PGH}$ rates $^{4}$ must be multiplied by a factor of two before comparison with the direct MD numerical simulations. The bare and PMF potentials at three different temperatures are shown in Fig. 4.

In the SBB model, both isomer wells and the potential barrier between them are approximated by a piecewise continuous parabolic potential which is solely defined by the barrier height $V^{+}$relative to the potential wells, the equilibrium position of the two isomers $\psi_{0}$ relative to the position of the barrier maximum, the barrier frequency $\omega^{+}$, and the frequencies at the minima of the potential wells, $\omega_{\mathrm{LiNC}}$ and $\omega_{\mathrm{LiCN}}$. The values of the parameters corresponding to the bare (viz., MEP) and effective (viz., PMF) potentials are summarized in Table III both for the forward and backward reactions.

The memory in the PGH model has been surmised through an exponential friction kernel $\zeta(t)$,

$$
\zeta(t)=\alpha^{-1} e^{-t / \alpha \gamma},
$$

TABLE III. Parameters in the PGH theory with the SBB potential approximation for the forward and backward reactions in the $\mathrm{LiNC} \rightleftharpoons \mathrm{LiCN}$ isomerization: $\omega^{+}, \omega_{\mathrm{LiNC}}$ and $\omega_{\mathrm{LiCN}}$ are the barrier and well frequencies, $\psi_{0}$ is the location of the well relative to the barrier, $V^{+}$is the barrier height, and $\alpha^{*}$ $=\alpha \omega^{+2}$ is the dimensionless parameter defining the bath friction kernel of the bare (MEP) and PMF potentials. All dimensional magnitudes are given in atomic units.

\begin{tabular}{lccccc}
\hline \hline LiNC $\rightarrow$ LiCN & $V^{+}$ & $\psi_{0}(\mathrm{rad})$ & $\omega^{+}$ & \multicolumn{1}{c}{$\omega_{\text {LiNC }}$} & $\alpha^{*}$ \\
\hline $\operatorname{MEP}$ & 0.0159 & 2.23 & $8.0 \times 10^{-4}$ & $4.4 \times 10^{-4}$ & 0.84 \\
$\operatorname{PMF}(T=2500 \mathrm{~K})$ & 0.0142 & 2.27 & $9.47 \times 10^{-4}$ & $3.83 \times 10^{-4}$ & 0.57 \\
$\operatorname{PMF}(T=3500 \mathrm{~K})$ & 0.0131 & 2.28 & $9.35 \times 10^{-4}$ & $3.71 \times 10^{-4}$ & 0.98 \\
$\operatorname{PMF}(T=5500 \mathrm{~K})$ & 0.0125 & 2.32 & $9.00 \times 10^{-4}$ & $3.48 \times 10^{-4}$ & 0.90 \\
& & & & & \\
$\mathrm{LiCN} \rightarrow \mathrm{LiNC}$ & $V^{+}$ & $\psi_{0}(\mathrm{rad})$ & $\omega^{+}$ & $\omega_{\mathrm{LiCN}}$ & $\alpha^{*}$ \\
\hline $\operatorname{MEP}$ & 0.005 & 0.91 & $8.0 \times 10^{-4}$ & $6.9 \times 10^{-4}$ & 0.84 \\
$\operatorname{PMF}(T=2500 \mathrm{~K})$ & 0.0044 & 0.87 & $9.47 \times 10^{-4}$ & $6.13 \times 10^{-4}$ & 0.57 \\
$\operatorname{PMF}(T=3500 \mathrm{~K})$ & 0.0041 & 0.85 & $9.35 \times 10^{-4}$ & $6.00 \times 10^{-4}$ & 0.98 \\
$\operatorname{PMF}(T=5500 \mathrm{~K})$ & 0.0035 & 0.82 & $9.00 \times 10^{-4}$ & $5.78 \times 10^{-4}$ & 0.90 \\
\hline \hline
\end{tabular}
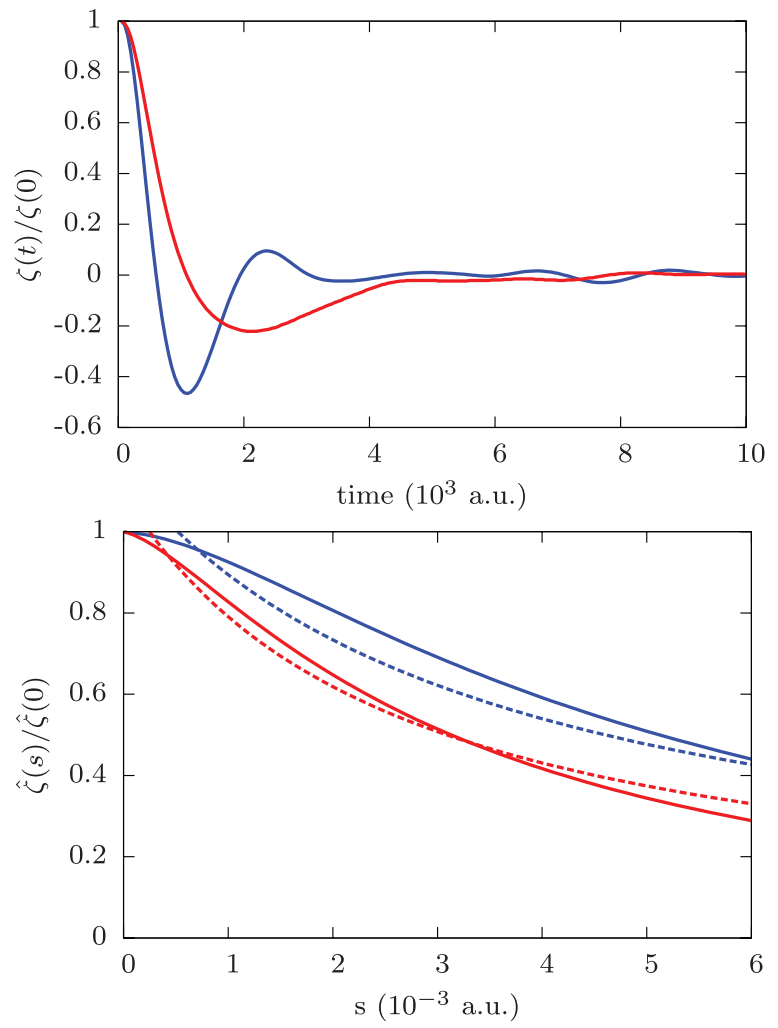

FIG. 5. Memory friction in the time (top) and Laplace (bottom) domains for bath densities of $45.5 \mathrm{~mol} / \mathrm{l}$ (red) and $89.5 \mathrm{~mol} / \mathrm{l}$ (blue). Best fits to Eq. (8) in the Laplace domain are shown as dashed curves in the bottom panel.

whose Laplace transform is

$$
\hat{\zeta}(s)=\frac{\gamma}{1+s \gamma \alpha},
$$

where $\gamma$ is the damping parameter, and $\alpha$ is the inverse of the infinite frequency shear modulus of the solvent. This last parameter has been adjusted to render a best fit to the memory function calculated by molecular dynamics simulations. As can be seen in Fig. 5, the Laplace transform of the friction memory function is reasonably well approximated by the function given by Eq. (8). The violations in the fit correspond to features at timescales of the order of $10^{3}$ a.u. These are much shorter than the timescales observed in the rates obtained below (with the fastest being on the order of $10^{5}$ a.u.), and hence the deviations from the simple exponential memory kernel can be safely ignored. All the parameters that are needed for the application of the PGH theory are summarized in Table III.

\section{Applicability of the Pollak-Grabert-Hänggi theory to $L i N C \rightleftharpoons$ LiCN isomerization}

The PGH theory stipulates one primary criterion that is sufficient for the derived reaction rate expression to be valid and give accurate results. ${ }^{4}$ Namely, $Q^{\ddagger} \gg k_{\mathrm{B}} T$, where $Q^{\ddagger}$ is the effective potential height, $Q^{\ddagger} \gg \Delta E$. The expression for computing the average energy loss $\Delta E$ may be found in Eq. (3.28) of Ref. 4. They also defined a perturbation parameter $\epsilon_{\mathrm{PGH}}$ as specified in Eqs. (2.19) and (2.20) of Ref. 4 which must be small in order for the harmonic approximation to hold. In the 

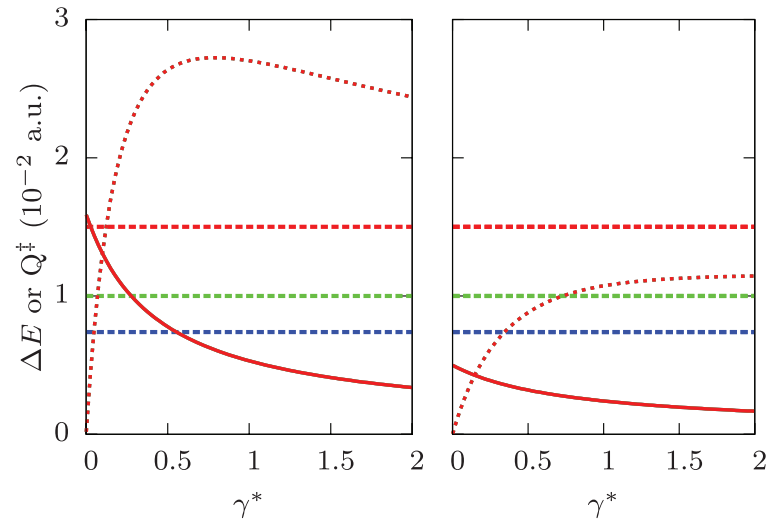

FIG. 6. Effective potential height $Q^{\ddagger}$ (red solid line), and average energy loss $\Delta E$ (red dashed line), as a function of the normalized bath viscosity, $\gamma^{*}=\gamma / \omega^{+}$, for the forward (left) and backward (right) reactions in the $\mathrm{LiNC} \rightleftharpoons \mathrm{LiCN}$ isomerization in an argon bath. (These are displayed relative to the bare MEP potential, and not the effective PMF, for simplicity in presentation as the former have no temperature dependence in the barrier height. The conclusions that can be drawn from the latter are essentially the same.) The values of the bath thermal energies corresponding to $T=2500 \mathrm{~K}$ (blue horizontal line), $3500 \mathrm{~K}$ (green horizontal line), and $5500 \mathrm{~K}$ (red horizontal line) are also shown. Recall that, according to Ref. $4, Q^{\ddagger} \gg k_{B} T$ in order for the PGH theory to be valid.

particular case of the SBB potential, which is used to represent $V_{\mathrm{PMF}}(\psi)$ as discussed above, we use the particular expression, Eq. (4.12), also derived in Ref. 4. Let us now check whether and when the PGH theory is expected to render good results in the calculation of the title reactions rate constants. For this purpose, we show in Fig. 6 the relevant magnitudes for the three temperatures considered in this work, as a function of the reduced bath friction parameter, $\gamma^{*}=\gamma / \omega^{+}$. We found that the parameter $\epsilon_{\mathrm{PGH}}$ is always smaller than 0.4 for the two reactions considered here. It is immediately apparent from the figures that the PGH criteria are only met for low values of $\gamma^{*}$, and even in this scenario they are satisfied only for the two lowest temperatures considered, i.e., $2500 \mathrm{~K}$ and $3500 \mathrm{~K}$. On the other hand, at $5500 \mathrm{~K}, Q^{\ddagger} \leq k_{\mathrm{B}} T$, and clearly violates the first validity condition for the applicability of the PGH theory. As will be seen below, the PGH rates are not in exact agreement with the MD results as expected from these considerations. However, they are in good qualitative agreement as can be justified from the degree to which these criteria are violated.

\section{Reaction rates in $\mathrm{LiNC} \rightleftharpoons \mathrm{LiCN}$ isomerization}

Rates for the forward and backward reactions implied in the $\mathrm{LiNC} \rightleftharpoons \mathrm{LiCN}$ isomerization in an argon bath have been calculated by classical MD simulations at the three temperatures that are been considered in this work. The density of the bath has been varied in such a way that the interaction between bath and isomerization reaction path, the energy diffusion and spatial diffusion regimes, and the Kramers turnover are explored. The corresponding results are shown in Fig. 7 and compared to the PGH model predictions. Three different temperatures, namely, $2500 \mathrm{~K}, 3500 \mathrm{~K}$ and $5500 \mathrm{~K}$, have been considered. The associated average thermal energies (as determined by energy equipartition over the corresponding
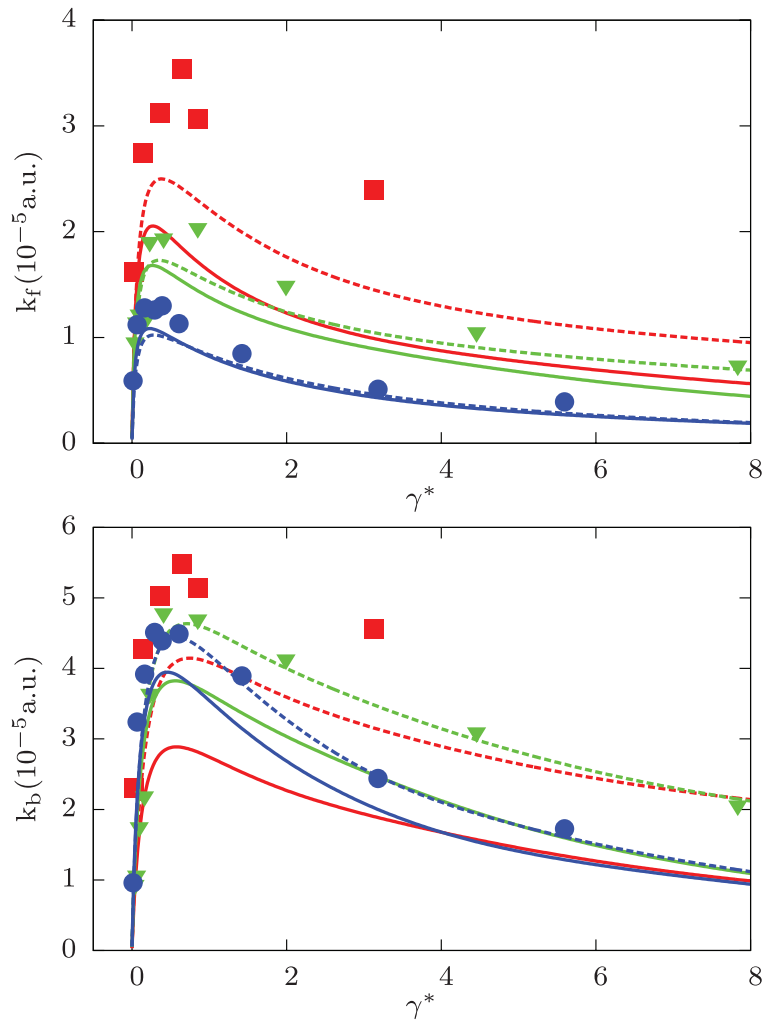

FIG. 7. Reaction rate for the forward (top) and backwards (bottom) $\mathrm{LiNC} \rightleftharpoons \mathrm{LiCN}$ isomerization in an argon bath as a function of the normalized viscosity, $\gamma^{*}=\gamma / \omega^{+}$, for three values of the temperature: $T=2500 \mathrm{~K}$ (blue), $3500 \mathrm{~K}$ (green), and $5500 \mathrm{~K}$ (red). Molecular dynamics calculation results are represented with colored squares, triangles, and circles. Colored curves give the Pollak-Grabert-Hänggi theory predictions using the bare (MEP) (solid line), and PMF (dashed line) potentials.

local minima) have been shown in Fig. 4. By examination of the corresponding energy profiles it can be ascertained that the forward rates are nominally in the activated regime with an average reactant energy lower than the barrier height. In the case of the backward rates, none of the three temperatures appear to be activated. Nevertheless, the rates for the lowest temperatures appear to follow an activated dynamics.

As can be seen in Fig. 7, in all cases, the forward reaction rates are lower than the backward rates, as can be expected from the different energy values of the associated barriers. It is also observed that the reaction rates increase with the bath friction in the energy diffusion regime $\left(\gamma^{*} \lesssim 0.2\right)$, until the Kramers turnover is reached, and this is the main and most important conclusion of our study. This effect is clearly visible in both reactions, although they obviously take place at different values of $\gamma^{*}$. Furthermore, the reaction rates start to decline when the bath viscosity (density) further increases, as expected in the spatial diffusion regime $\left(\gamma^{*} \gtrsim 0.5\right)$.

Furthermore, when the MD results are compared with those obtained with the PGH theory, the agreement found is consistent with the discussion in Subsection III B 2 on the applicability of the PGH theory to the title reactions. Indeed, at high temperatures, the PGH results are in poor agreement with the numerical MD rates. At the two lower temperatures and in the low friction regime, however, the rates are in good agreement. This is consistent with the fact 
that the theory-both with respect to the applicability of the reduced-dimensional model and the PGH rates obtained for said model-is more applicable as the governing inequalities are either satisfied or close to being so. Meanwhile, in the high friction regime at the two lower temperatures, we find good agreement between the theory and MD. This is perhaps surprising because the criteria detailed above were more strongly violated therein. However, the agreement stems from the fact that the rate at high friction is dominated by spatial diffusion and hence even large violations of the approximations enforced by these criteria lead to negligible corrections of the rates with respect to those obtained from the dynamical simulations.

\section{Further confirmation of the turnover}

One possible objection to the turnover observed in Fig. 7 and initially reported in Ref. 19 is the possibility that temperature may be changing with the associated $\gamma^{*}$. In the latter case, it is known ${ }^{18}$ that the rates will rise and fall not because of a true turnover through the dissipative regimes, but rather because the high temperatures lead to entropic bottlenecks that lead in turn to decreasing rates. Indeed, it is seen in the actual data for the forward reaction in Fig. 7 that the PGH rates at the highest $(5500 \mathrm{~K})$ and lowest temperature $(2500 \mathrm{~K})$ are both smaller in comparison with those at the intermediate temperature $(3500 \mathrm{~K})$. To further investigate this possibility and confirm our finding of a true Kramer turnover on the title reactions we computed the effective bath temperature during the simulations. Recall that temperature stability is guaranteed in our calculations through the use of the conventional thermostat, i.e., velocity rescaling of the solvent atoms, during the equilibration regime. However, during the calculation phase the system evolves freely conserving the total energy, temperature, and volume. Results are shown in Fig. 8. As can be seen, the observed temperatures are nearly flat for all the range of viscosities $\gamma^{*}$ considered, and they always corresponded to the temperature imposed. As a result, we can undoubtedly conclude that the observed turnover effect is not due to a variation of the temperature, but rather to be a true effect due to friction, as first reported.

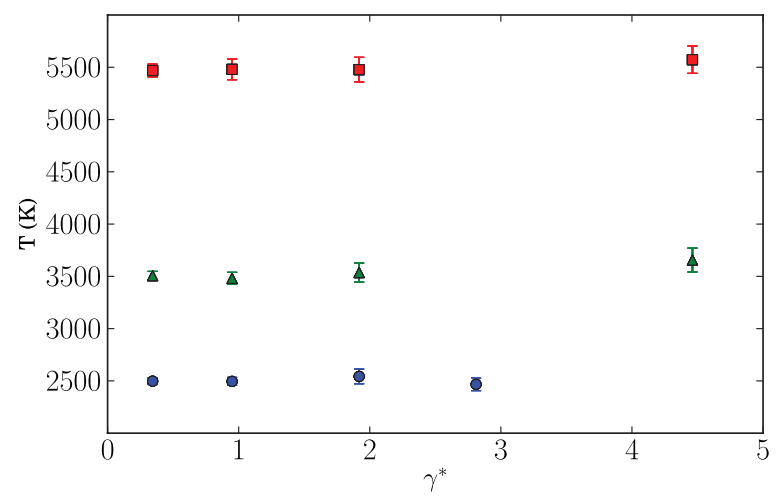

FIG. 8. Numerically measured temperature in the simulations of the $\mathrm{LiNC} \rightleftharpoons \mathrm{LiCN}$ isomerization reaction in an argon bath at same of the values of the effective viscosity, $\gamma^{*}$, and imposed temperature considered in Fig. 7.
The PGH theory predictions for the rates are in good agreement with the molecular dynamics simulations at the low and intermediate temperatures as shown in Fig. 7. In all cases, the rates are computed following Eq. (3.32) in Ref. 4 taking the one-dimensional potential either as the PMF (as one should) or the bare MEP (for comparison). Both sets of rates are displayed in the accompanying figures. At the lowest temperature, where the PGH theory should work best, it does, and it does so for rates obtained for the PMF. Even when PGH theory does not agree with the observed rates at the high temperatures, it still appears to follow the turnover behavior. This result, not reported in our earlier Ref. 19, indicates that both the forward and backward rates are determined by activated processes that are energy-diffusion limited at low friction and space-diffusion limited at high friction. Such is the case despite the fact that the thermal energy is near or exceeds the potential barriers along the one-dimensional reactive coordinate as indicated in Fig. 4. The activated dynamics must, therefore, be taking place in a higher dimensional reduced space suggesting that the one-dimensional approximation is insufficient. Indeed, the specific trajectories (not shown) indicate that the $R$-mode appears to be strongly coupled to the reaction coordinate-dominated $\psi$-mode. Such calculations can be performed, even for systems with classically chaotic dynamics, by using, for example, the approach developed in Ref. 26. Nevertheless, the key point is that a Kramers turnover has been observed for both the forward and backward $\mathrm{LiCN}$ reaction as a function of friction at three different temperatures.

\section{CONCLUSION}

In this work, we have numerically calculated the rates for the forward and backward reactions in the $\mathrm{LiNC} \rightleftharpoons \mathrm{LiCN}$ isomerization in an argon bath using explicit-atom MD simulations. According to our results, which have been thoroughly checked, both reaction rates exhibit the expected qualitative turnover across the dissipative regimes predicted by Kramers, and they agrees quantitatively with the rates of obtained from reduced-dimensional Langevin models, when the corresponding conditions for applicability are fulfilled. The latter were obtained using the Pollak-Grabert-Hänggi theory using a suitable Straub-Borkovec-Berne one-dimensional potential (viz., a continuous piecewise-parabolic approximation to the minimum energy path), and a memory represented by an exponential friction kernel.

To achieve convergence in the explicit-atom MD simulations of this study, a relatively high temperature has been employed. In order to explore lower temperatures, the use of rate formulas (for the direct dynamics) that have faster convergence without requiring substantially more computer resources should be used. In this direction, it may be possible to apply accelerated dynamics, ${ }^{27}$ transition path sampling, ${ }^{28}$ or the moving transition state approach. ${ }^{29,30}$ Nevertheless, the success of the model chemical reactive system at reproducing the Kramers turnover also makes one hopeful that it might reveal the extent to which the neglect or incorporation of stochastic accelerations is important in the choice of coordinate system. ${ }^{31}$ 


\section{ACKNOWLEDGMENTS}

This work has been supported by the Spanish MINECO under projects MTM2009-14621 and ICMAT Severo Ochoa SEV-2011-0087. It also has been partially supported by the U.S. National Science Foundation under Grant No. CHE-1112067. Travel between partners was partially supported through the People Programme (Marie Curie Actions) of the European Union's Seventh Framework Programme FP7/2007-2013/ under REA Grant Agreement No. 294974.

${ }^{1}$ R. Brown, Philos. Mag. 4, 161 (1828).

${ }^{2}$ H. Kramers, Physica (Utrech) 7, 284 (1940).

${ }^{3}$ V. I. Mel'nikov and S. V. Meshnov, J. Chem. Phys. 85, 1018 (1986).

${ }^{4}$ E. Pollak, H. Grabert, and P. Hänggi, J. Chem. Phys. 91, 4073 (1989).

${ }^{5}$ K. Lindenberg and E. Cortés, Physica A 126, 489 (1984).

${ }^{6}$ E. Pollak, J. Chem. Phys. 85, 865 (1986).

${ }^{7}$ E. Pollak, S. C. Tucker, and B. J. Berne, Phys. Rev. Let. 65, 1399 (1990).

${ }^{8}$ E. Pollak and P. Talkner, Phys. Rev. E 51, 1868 (1995).

${ }^{9}$ K. Lindenberg, A. H. Romero, and J. M. Sancho, Physica D 133, 348 (1999).

${ }^{10}$ E. Pollak and P. Talkner, Chaos 15, 026116 (2005).

${ }^{11}$ R. Hernandez, T. Bartsch, and T. Uzer, Chem. Phys. 370, 270 (2010).

${ }^{12}$ S. Abrash, S. Repinec, and R. M. Hochstrasser, J. Chem. Phys. 93, 1041 (1990).

${ }^{13}$ L. Nikowa, D. Schwarzer, J. Troe, and J. Schoeder, J. Chem. Phys. 97, 4827 (1992).
${ }^{14}$ J. Ashcroft, C.-L. Xie, and J. Jonas, J. Chem. Phys. 90, 5386 (1989).

${ }^{15}$ C.-L. Xie, D. Campbell, and J. Jonas, J. Chem. Phys. 92, 3736 (1990).

${ }^{16}$ A. J. Marks, J. N. Murrel, and A. J. Stace, Chem. Phys. Lett. 154, 492 (1989).

${ }^{17}$ A. Lopez-Rubio and J. M. Lagaron, Food Chem. 125, 997 (2011).

${ }^{18}$ B. J. Berne, M. Borkovec, and J. E. Straub, J. Phys. Chem. 92, 3711 (1988).

${ }^{19}$ P. García-Müller, R. Hernandez, R. M. Benito, and F. Borondo, Phys. Rev. Lett. 101, 178302 (2008).

${ }^{20}$ J. Perkins, E. Edwards, R. Kleiv, and N. Weinberg, Mol. Phys. 109, 1901 (2011).

${ }^{21}$ R. Essers, J. Tennyson, and P. E. S. Wormer, Chem. Phys. Lett. 89, 223 (1982).

${ }^{22}$ J. E. Straub, M. Borkovec, and B. J. Berne, J. Chem. Phys. 89, 4833 (1988).

${ }^{23}$ J. E. Straub, M. Borkovec, and B. J. Berne, J. Phys. Chem. 83, 3172 (1985).

${ }^{24}$ W. H. Miller, N. C. Handy, and J. E. Adams, J. Chem. Phys. 72, 99 (1980).

${ }^{25}$ D. A. McQuarrie, Statistical Mechanics (University Science Books, Sausalito, CA, 2000).

${ }^{26}$ E. Hershkovitz and L. Wiesenfeld, J. Chem. Phys. 113, 4558 (2000).

${ }^{27}$ A. F. Voter, F. Montalenti, and T. C. Germann, Annu. Rev. Mater. Res. 32, 321 (2002).

${ }^{28}$ P. G. Bolhuis, D. Chandler, C. Dellago, and P. Geissler, Annu. Rev. Phys. Chem. 53, 291 (2002).

${ }^{29}$ T. Bartsch, R. Hernandez, and T. Uzer, Phys. Rev. Lett. 95, 058301 (2005).

${ }^{30}$ T. Bartsch, T. Uzer, J. M. Moix, and R. Hernandez, J. Phys. Chem. B 112, 206 (2008).

${ }^{31}$ J. M. Moix, R. Hernandez, and E. Pollak, J. Phys. Chem. B 112, 213 (2008). 